\title{
Competing Wave-Breaking Mechanisms in Quadratic Media
}

\author{
Matteo Conforti ${ }^{1}$, Fabio Baronio $^{1}$, and Stefano Trillo ${ }^{2}$ \\ ${ }^{1}$ CNISM, Dipartimento di Ingegneria dell'Informazione, Università di Brescia, Via Branze 38, 25123 Brescia, Italy \\ ${ }^{2}$ Dipartimento di Ingegneria, Università di Ferrara, Via Saragat 1, 44122 Ferrara, Italy
}

Compiled March 24, 2013

\begin{abstract}
We show that second-harmonic generation in the regime of weak dispersion/diffraction can exhibit a coexistence of wave breaking mechanisms, such that a gradient catastrophe yielding a dispersive shock wave competes with modulational instability, leading to the generation of wavetrains with incommensurate frequencies, and eventually to destruction of the shock wave-train. (C) 2013 Optical Society of America

OCIS codes: 000.0000, 999.9999.
\end{abstract}

Dispersive nonlinear wave propagation give rise to different universal mechanisms of breaking. Modulational (or Bejamin-Feir-Lighthill) instability (MI), in its basic manifestation, entails the breaking of a carrier wave due to growth of low frequency modulations [1-3]. A different mechanism involves, in the weakly dispersive (diffractive) limit, a gradient catastrophe, where a smooth envelope steepens until it develops an infinite gradient at finite distance. This behavior is characteristic of Hamiltonian systems which possess a hyperbolic dispersionless limit. After the breaking (catastrophe) point, unsteady fast oscillations appear due to the regularizing action played by the dispersion, forming what is called a dispersive shock waves (DSW) [4-9]. For the scalar nonlinear Schrödinger (NLS) equation, describing Kerr media, these two mechanims are mutually exclusive. In fact, the gradient catastrophe occurs in the defocusing regime characterized indeed by a hyperbolic dispersionless limit [5-9]. Conversely, MI takes place in the focusing regime where the gradient catastrophe is precluded, reflecting the elliptic dispersionless limit of this case.

In this letter we show that, when considering second harmonic generation (SHG), the two mechanisms may coexist inducing a new scenario where the onset of MI $[10,11]$ dramatically affects a two-color DSW emanating from a gradient catastrophe occurring on both fields. In order to analyze such competition, we consider SHG in a regime of low (or even zero) mismatch, establishing the fact that DSW do not require to operate in the cascading (high mismatch) regime, where SHG mimics the dynamics of Kerr media [12]. We start from the 1+1D model for type I SHG in a planar waveguide $[13,14]$ with weak diffraction

$$
\begin{gathered}
i \varepsilon u_{1 z}+\beta_{1} \frac{\varepsilon^{2}}{2} u_{1 x x}+u_{2} u_{1}^{*}=0, \\
i \varepsilon u_{2 z}+\beta_{2} \frac{\varepsilon^{2}}{2} u_{2 x x}-\delta k u_{2}+u_{1}^{2}=0,
\end{gathered}
$$

where $u_{1}(z, x)$ and $u_{2}(z, x)$ are normalized envelopes at fundamental frequency $(\mathrm{FF})$ and its second harmonic (SH), respectively, $x$ is the transverse coordinate in units of beam width $W_{0}, z$ is the distance in units of $L=\sqrt{L_{D} L_{N L}}, L_{N L}=1 /(\chi \sqrt{P})$ and $L_{D}=k_{1} W_{0}^{2}$ being the nonlinear and diffraction lengths associated with nonlinearity $\chi$, power $P$ and beam width $W_{0}$. The parameter $\varepsilon=\sqrt{L_{N L} / L_{D}}$ sets the weakness of diffraction. $\delta k=\left(2 k_{1}-k_{2}\right) L_{N L}$ is the mismatch, with $\beta_{j}=k_{j} / k_{1}$, $k_{1,2}$ being $\mathrm{FF}$ and $\mathrm{SH}$ wavenumbers.

The competition of wave-breaking mechanism can be efficiently investigated by restricting to the experimentally accessible case [15] of FF and $\mathrm{SH}$ phase-locked beams. In the limit of vanishing diffraction, strict phase-locking at any $z$ can be satisfied only for nonlinear eigenmodes of Eqs. (1-2) [10], i.e. plane waves $u_{1}(z)=U_{1} \exp (i s \mu z / \varepsilon), u_{2}(z)=s U_{2} \exp (i 2 s \mu z / \varepsilon)$, which are solutions of Eqs. (1-2) provided that $U_{1}= \pm \sqrt{\mu(2 \mu+s \delta k)}, \quad U_{2}=\mu$, where $\mu>0$ is arbitrary and the $\mathrm{SH}$ sign $s= \pm 1$ is conveniently introduced to discriminate between in-phase $(s=1$, $\phi=0)$ and out-of-phase $(s=-1, \phi=\pi)$ locking of the overall phase $\phi=\phi_{2}-2 \phi_{1}$, with $\phi_{1,2} \equiv \arg \left(u_{1,2}\right)$. Since plane-waves give rise to MI but not to shock formation, we show that the competition phenomenon can be conveniently addressed by considering the evolution of quasi-eigenmodes, i.e. beams with arbitrary $x$-shape which evolve while maintaining valid only locally (both in $x$ and $z$ ) the eigenmode constraint. Our aim is to develop a simple hydrodynamic description of the evolution of such beams, which is valid under the hypothesis that they remain phase-locked upon evolution (a general hydrodynamic reduction of Eqs. (1-2) remains a challenging open problem, which is beyond the scope of this paper). To this end we apply to Eqs. (1-2) the Madelung (or WKB) transformation $u_{1}(z, x)=\sqrt{\rho_{1}(z, x)} \exp \left[i S_{1}(z, x) / \varepsilon\right]$, $u_{2}(z, x)=s \sqrt{\rho_{2}(z, x)} \exp \left[i S_{2}(z, x) / \varepsilon\right]$, assuming $S_{2}(z, x)=2 S_{1}(z, x)$. Neglecting terms $O\left(\varepsilon^{2}\right)$, we obtain the first-order quasi-linear system of equations

$$
\begin{aligned}
& \rho_{1 z}+\beta_{1}\left(v_{1} \rho_{1}\right)_{x}=0, \quad \rho_{2 z}+\beta_{2}\left(v_{2} \rho_{2}\right)_{x}=0, \\
& \left(1+\frac{\rho_{1}}{2 \rho_{2}}\right) v_{z}+\left(2 \beta_{2}+\beta_{1} \frac{\rho_{1}}{2 \rho_{2}}\right) v v_{x}-\frac{s}{2} \frac{\rho_{1 x}}{\sqrt{\rho_{2}}}=0 .
\end{aligned}
$$

where $\rho_{1,2}$ and $v_{1,2} \equiv S_{1,2}$ play the role of equivalent densities and velocities of two coupled fluids [4]. Note, however, that the first two of Eqs. (3) entail no direct coupling terms between the two densities, equivalent to transfer of mass, as a consequence of the phase-locking assumption. We have reduced the system of four equations for the unknowns $\left(\rho_{1}, \rho_{2}, v_{1}, v_{2}\right)$ just to three by imposing the compatibility constraint $v_{1}=v_{2} / 2 \equiv v$. Following the quasi-eigenmode hypothesis, we assume 

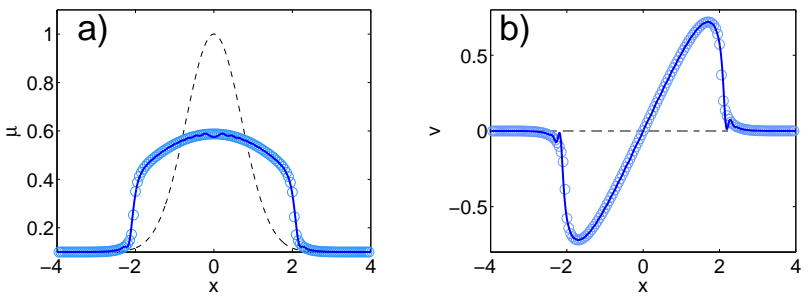

Fig. 1. (Color online) (a) Equivalent fluid density $\mu$ and (b) velocity $v$ at distance $z=1.4 \simeq z_{s}$ from numerical integration of Eqs. (5) with $\delta k=-4$ (solid blue curves), compared with SH amplitude $\left|u_{2}\right|$ and chirp $S_{2 x}$ from Eqs. (1-2) with $\varepsilon=0.05$ (dots), and input (dashed line).

$\rho_{1,2}=\rho_{1,2}(z, x)$ to vary in such a way to follow locally the eigenmode constraint, so that:

$$
\rho_{1}=\mu(z, x)[2 \mu(z, x)+s \delta k], \quad \rho_{2}=\mu^{2}(z, x) .
$$

The first two of Eqs. (3) are continuity equations that represent the conservation in differential form of the individual FF and $\mathrm{SH}$ powers, that hold strictly only for plane-wave eigenmodes. However, since we allow $\mu$ to vary with $z, \mathrm{FF}$ and $\mathrm{SH}$ no longer strictly conserve their powers independently. However, conservation must hold for total power, and we can derive a continuity equation for the total density $\rho=\rho_{1}+\rho_{2}=\mu(3 \mu+s \delta k)$, by inserting Eqs. (4) into the the first two of Eqs. (3), and summing up. Moreover we exploit the relation $\beta_{2}=\beta_{1} / 2=1 / 2$, thus obtaining $\rho_{z}+(v \rho)_{x}=0$ and $v_{z}+v v_{x}-s \mu_{x}=0$, which, once further cast in terms of the "fluid density" $\mu$ (SH amplitude), read as

$$
\mu_{z}+(v \mu)_{x}-g(\mu) \mu v_{x}=0 ; \quad v_{z}+v v_{x}-s \mu_{x}=0 .
$$

Equations (5) constitute a reduced hydrodynamic description, which is reminiscent of the dispersionless limit of the NLS [4], except for an extra correction term weighted by the function $g(\mu) \equiv 3 \mu /(6 \mu+s \delta k)$, which, depending on the value of $\delta k$, can take values in the range $[0,1 / 2]$. However, two important differences must be emphasized: (i) Since the Riemann eigenvelocities associated with Eqs. (5) are $V^{ \pm}=v \pm \sqrt{-s(1-g) \mu}$, the SHG interaction allows to investigate in the same physical system both the elliptic problem $\left(s=1, V^{ \pm}\right.$are c.c.) and the hyperbolic problem $\left(s=-1, V^{ \pm}\right.$are real), by simply flipping the locking of the phases of the FF and $\mathrm{SH}$, which can be easily accomplished experimentally [15]; (ii) although Eqs. (5) are reminiscent of the NLS dispersionless limit, they do not require to operate in the high-mismatch regime (cascading), where SHG is approximated by the NLS equation [14]. This limit, studied in Ref. [12] is correctly recovered for $|\delta k| \rightarrow \infty$, which yields $g=0$. However Eqs. (5) hold valid under much more general conditions, also when the genuine nature of the parametric process is retained, namely for perfect phase matching $\delta k=0$, that gives $g=1 / 2$. In the following we focus on the hyperbolic case $s=-1$ $(\phi=\pi)$ and its gradient catastrophe. While Eqs. (5) entail breaking for a variety of smooth input, in order to be specific we consider common launching conditions
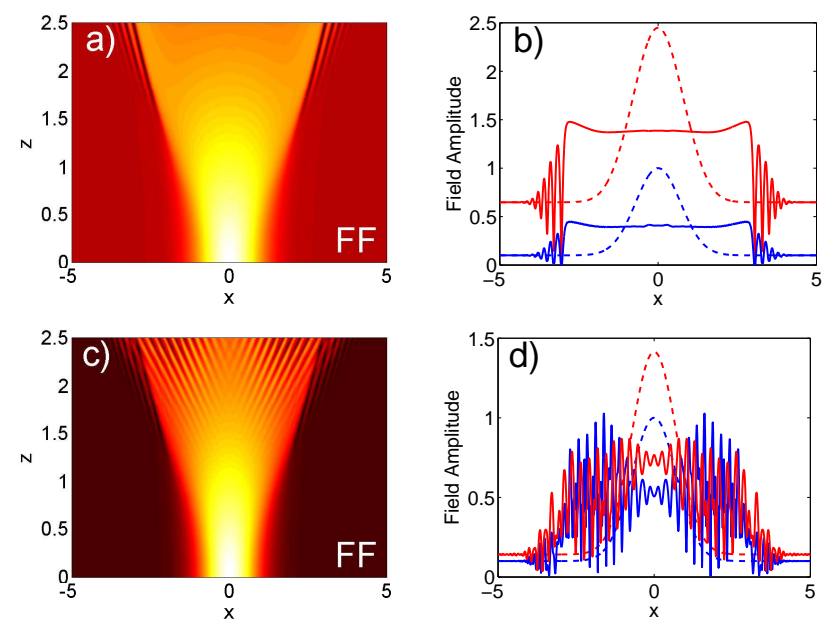

Fig. 2. (Color online) (a,c) Color level plot of FF field $\left|u_{1}\right|$, ruled by Eqs. (1-2) (the SH, not shown, is similar); (b,d) Snapshots of output amplitudes $\left|u_{1}\right|$ (FF, solid red) and $\left|u_{2}\right|$ (SH, solid blue). Dashed curves are the input. Here $\varepsilon=0.05$ and $\delta k=-4(\mathrm{a}, \mathrm{b})$ or $\delta k=0(\mathrm{c}, \mathrm{d})$.

used in experiments [6], i.e. unchirped Gaussian beams on pedestal: $\mu(0, x)=\nu+(1-\nu) \exp \left(-x^{2}\right), v(0, x)=0$, with $\nu \ll 1$ (for definiteness, examples are reported for $\nu=0.1$ ). According to numerical solutions of Eqs. (5), breaking occurs via two symmetric gradient catastrophes at finite distance $z_{s}$ (which slightly depends on $\delta k$ ). This is displayed in Fig. 1 for $\delta k=-4\left(z_{s}=1.4\right)$ and compared with simulation of the full dynamics ruled by Eqs. (1-2) from input $u_{1}(0, x)=\sqrt{\mu(0, x)[2 \mu(0, x)-\delta k]}$, $u_{2}(0, x)=-\mu(0, x)$. The reduced system (5) is found to capture the breaking dynamics with remarkable accuracy in a wide range of $\delta k$. We have also verified (data not shown) that the phases remain nearly locked to yield $\phi \simeq \pi$ in spite of their large individual changes with $z$.

We then proceed to study the dynamics beyond the critical point $z_{s}$, where the validity of Eqs. (5) breaks down. In particular our aim is to show that, changing the mismatch $\delta k$, severely and qualitatively affects the post-shock dynamics. We show examples obtained from numerical integration of the original model (1-2) for fixed $\varepsilon=0.05$ The SHG dynamics for $\delta k=-4$ is illustrated in Fig. 2(a,b). The weak diffraction regularizes the shocks (steep fronts in Fig. 1) originating from the gradient catastrophe, through the appearance of two DSW which fill with fast oscillations extending portions of space in outward directions, well visible in the snapshots of the output fields in Fig. 2(b). However, the scenario changes qualitatively at phase-matching $(\delta k=0)$ as illustrated in Fig. 2(c,d). While the emergence of the DSW is similar compared to the mismatched case, independent additional oscillations with incommensurate frequency appear on top of the beams, inducing a loss of coherence of the DSW, thus making the behavior rather chaotic already at relatively short distance $[z=2.5$, see Fig. 2(d)]. This behavior, which bears no similarity in experiments performed on DSW and described by defocusing NLS-type models $[5-7,9]$, must be ascribed to the onset of MI. The latter arises when pe- 

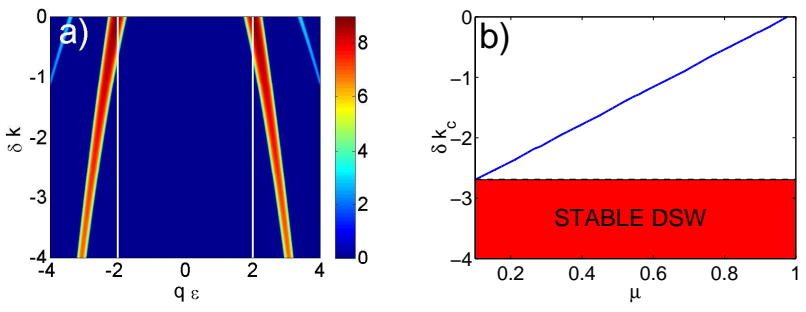

Fig. 3. (Color online) (a) Color level plot of MI gain vs. scaled wavenumber $\varepsilon q$ and phase mismatch $\delta k$, for $\mu=1$. The intersecting white line indicates the estimated wavenumber $q_{S H}$ from the DSW; (b) Critical mismatch $\delta k_{c}$ [value at intersection between $q_{S H}$ and the gain bandwith in (a)] vs. $\mu$. The shaded (red) region gives stable DSW.
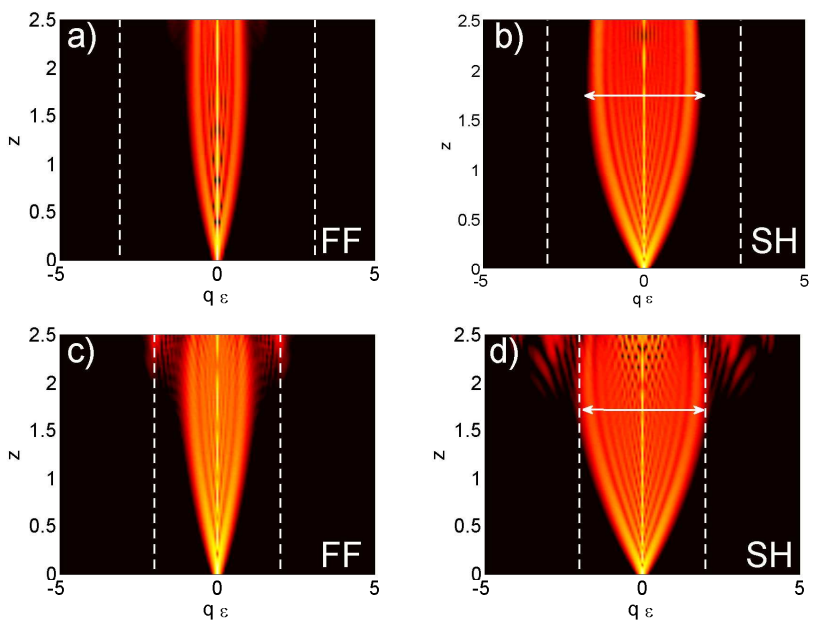

Fig. 4. (Color online) Evolution of spatial spectra for stable DSW at $\delta k=-4[(\mathrm{a}) \mathrm{FF}$; (b) SH] and competing $\mathrm{MI}$ at $\delta k=0[(\mathrm{c}) \mathrm{FF}$; (d) $\mathrm{SH}]$. The double arrow indicates the estimated DSW frequency $q_{S H}$ (see text). The dashed vertical lines refers to the peak of MI gain $g(q)$.

riodic perturbations with wavenumber $q$, in this case $p_{m}(z, x)=a_{m}(z) \exp [i q x]+b_{m}(z) \exp [-i q x], m=1,2$, grow exponentially like $\exp (g z)$ at the expense of the pump beams. The gain $g(q)$ and its bandwidth are obtained from the most unstable eigenvalue of the standard linearized problem for the perturbation. The outcome of such analysis [10] (see also review papers [13,14]) remains valid under the semiclassical scaling of Eqs. (12 ) provided the rescaling $g(q) \rightarrow \varepsilon^{-1} g(\varepsilon q)$ is adopted. Here we show that such analysis quantitatively explains the competition mechanism, even though we apply it to slowly evolving fields instead of strict plane-wave eigenmodes. The argument is as follows: the gradient catastrophe is accompanied by a dramatic spectral broadening which is stabilized when the DSW starts to develop. Under this condition a good measure of the spectral peak edge associated with the modulated periodic wave at $\mathrm{SH}$ turns out to be $q_{S H} \approx 2 / \varepsilon$. The onset of MI is expected whenever $q_{S H}$ falls under the MI gain bandwidth, shown in Fig. 4(a) as a function of $\delta k$ for $\mu=1$. Overlap be- tween $q_{S H}$ and $g(q)$ clearly occurs at $\delta k=0$. By studying how this overlap behaves with $\mu$ (MI bandwidth shifts at lower $q$ for decreasing amplitude $\mu$ ), we end up with a critical $\delta k_{c}=\delta k_{c}(\mu)$ reported as a curve in Fig. 3(b). In order to avoid the overlap (and hence MI) for the whole beam (range of values $\mu=0.1-1$ ), one has to operate below the whole curve, i.e. at $\delta k<-3$ [shaded region in Fig. 4(b)], as confirmed by our numerics [see Fig. 2]. The evolution of the spectra reported in Fig. 4 further confirm this interpretation. When DSW are stable, no spectral components are generated in the vicinity of the spectral peak of $g(q)$ [see Fig. 4(a-b)]. Viceversa, as shown in Fig. 4(c-d) for $\delta k=0$, the onset of MI is clearly due to the spectral $\mathrm{SH}$ wavenumbers (estimated by $q_{S H}$, and reported as a double arrow) which hit the MI gain peak (dashed line), thus leading such components to be strongly amplified simultaneously in both FF and SH modes, as clearly visible in Fig. 4(c-d). Finally we point out that, for smaller values of $\varepsilon(\sim 0.01)$, the MI can build up from noise even at distances shorter than the characteristic shock distance, thus dominating the dynamics.

In summary, SHG supports a novel dynamical scenario where two-color DSW formed via gradient catastrophes are deeply affected by MI. This suggests that the concomitance of two wave-breaking mechanisms is a general feature for modes which are nonlinearly coupled [16].

Funding from MIUR (grant PRIN 2009P3K72Z) are gratefully acknowledged.

\section{References}

1. M.J. Lighthill, J. Inst. Math. Appl. 1, 269 (1965).

2. T.B. Benjamin and J.E. Feir, J. Fluid Mech. 27, 417 (1967).

3. K. Tai, A. Hasegawa, and A. Tomita, Phys. Rev. Lett. 56, 135 (1986).

4. A.V. Gurevich and A. L. Krylov, Sov. Phys. JETP 65, 944 (1987).

5. J. E. Rothenberg and D. Grischkowsky, Phys. Rev. Lett. 62, 531 (1989).

6. W. Wan, S. Jia, and J. W. Fleischer, Nature Phys. 3, 46 (2007).

7. N. Ghofraniha, C. Conti, G. Ruocco, S. Trillo, Phys. Rev. Lett. 99, 043903 (2007).

8. S. Jia, W. Wan, and J. W. Fleischer, Phys. Rev. Lett. 99, 223901 (2007).

9. C. Conti, A. Fratalocchi, M. Peccianti, G. Ruocco, and S. Trillo, Phys. Rev. Lett. 102, 083902 (2009).

10. S. Trillo and P. Ferro, Opt. Lett. 20, 438 (1995).

11. R. A. Fuerst, D. M. Baboiu, B. Lawrence, W. E. Torruellas, G. I. Stegeman, S. Trillo, and S. Wabnitz, Phys. Rev. Lett. 78, 2756 (1997).

12. M. Conforti, F. Baronio, and S. Trillo, Opt. Lett. 37, 1082 (2012).

13. C. Etrich, F. Lederer, B. A. Malomed, T. Peschel, and U. Peschel, Progr. Optics 41, 483 (2000).

14. A. V. Buryak, P. Di Trapani, D. V. Skryabin, and S. Trillo, Phys. Rep. 370, 63 (2002).

15. W. E. Torruellas, Z. Wang, D. J. Hagan, E. W. VanStryland, and G. I. Stegeman, Phys. Rev. Lett. 74, 5036 (1995).

16. J. E. Rothenberg, Opt. Lett. 16, 18 (1991). 


\section{References}

1. M.J. Lighthill, "Contribution to the theory of waves in nonlinear dispersive systems", J. Inst. Math. Appl. 1, 269 (1965).

2. T.B. Benjamin and J.E. Feir, "The disintegration of wavetrains on deep water. Part 1: Theory," J. Fluid Mech. 27, 417 (1967).

3. K. Tai, A. Hasegawa, and A. Tomita, "Observation of modulation instability in optical fibers," Phys. Rev. Lett. 56, 135 (1986).

4. A.V. Gurevich and A. L. Krylov, "Dissipationless shock waves in media with positive dispersion", Sov. Phys. JETP 65, 944 (1987);

5. J. E. Rothenberg and D. Grischkowsky, "Observation of the formation of an optical intensity shock and wavebreaking in the nonlinear propagation of pulses in optical fibers", Phys. Rev. Lett. 62, 531 (1989).

6. W. Wan, S. Jia, and J. W. Fleischer, "Dispersive superfluid-like shock waves in nonlinear optics", Nature Phys. 3, 46 (2007).

7. N. Ghofraniha, C. Conti, G. Ruocco, S. Trillo, "Shocks in nonlocal media", Phys. Rev. Lett. 99, 043903 (2007).

8. S. Jia, W. Wan, and J. W. Fleischer, "Dispersive shock waves in nonlinear arrays", Phys. Rev. Lett. 99, 223901 (2007).

9. C. Conti, A. Fratalocchi, M. Peccianti, G. Ruocco, and S. Trillo, "Observation of a gradient catastrophe generating solitons", Phys. Rev. Lett. 102, 083902 (2009).

10. S. Trillo and P. Ferro, "Modulational instability in second-harmonic generation", Opt. Lett. 20, 438 (1995).

11. R. A. Fuerst, D. M. Baboiu, B. Lawrence, W. E. Torruellas, G. I. Stegeman, S. Trillo, and S. Wabnitz, "Spatial modulational instability and multisoliton-like generation in a quadratically nonlinear optical medium", Phys. Rev. Lett. 78, 2756 (1997).

12. M. Conforti, F. Baronio, and S. Trillo, "Dispersive shock waves in phase-mismatched second harmonic generation", Opt. Lett. 37, 1082 (2012).

13. C. Etrich, F. Lederer, B. A. Malomed, T. Peschel, and U. Peschel, "Optical solitons in media with a quadratic nonlinearity", Progr. Optics 41, 483 (2000).

14. A. V. Buryak, P. Di Trapani, D. V. Skryabin, and S. Trillo, "Optical solitons due to quadratic nonlinearities: from basic physics to futuristic applications", Phys. Rep. 370, 63 (2002).

15. W. E. Torruellas, Z. Wang, D. J. Hagan, E. W. VanStryland, and G. I. Stegeman, "Observation of TwoDimensional Spatial Solitary Waves in a Quadratic Medium", Phys. Rev. Lett. 74, 5036 (1995).

16. J. E. Rothenberg, "Observation of the buildup of modulational instability from wave breaking", Opt. Lett. 16, 18 (1991). 